Review

\title{
Beauvericin: the journey of a pesticide into a humanized drug
}

\author{
Charbel Al Khoury ${ }^{1, *}$, Nabil Nemer ${ }^{2}$, Sima Tokajian ${ }^{1}$, and Georges Nemer ${ }^{3,4, *}$ \\ 1 Department of Natural Sciences, School of Art and Sciences, Lebanese American University, Byblos Cam- \\ pus, P.O. Box 36, Byblos, Lebanon \\ 2 Department of Agriculture and Food Engineering, Holy Spirit University of Kaslik, P.O. Box 446, Jounieh, \\ Lebanon \\ 3 Department of Biochemistry and Molecular Genetics, Faculty of Medicine, American University of Beirut, \\ P.O. Box 110236 Beirut, Lebanon \\ 4 Division of Genomics and Translational Biomedicine, College of Health and Life Sciences, Hamad Bin Kha- \\ lifa University, P.O. Box 34110 Doha, Qatar \\ * GNemer@hbku.edu.qa; Charbel.alkhoury@lau.edu.lb
}

\begin{abstract}
Drug discovery has been initially attributed to coincidence or trial and error where the traditional approach was complex, lengthy, and expensive. Conventional drug discovery methods require the costly random screening of synthesized compounds or natural products. Another downside for this approach is the wide dependency on the experimental use of animals for in vivo testing. Currently, in silico modeling has become a vital tool for drug discovery and repurposing, and molecular docking is being used to find the best matching between a ligand and a molecule. Practical application of in silico docking will predict the biomolecular interactions between the drug and the target host. Beauvericin (BEA) is an emerging mycotoxin produced by the entomopathogenic fungus Beauveria bassiana. Originally investigated for its pesticide capability, BEA is now considered as a molecule of interest for its potentially diverse biotechnological applications in the pharmacological industry and the field of medicine. In this manuscript we will provide an overview on the repurposing of BEA into a potentially superior therapeutic molecule in a broad range of diseases. Furthermore, a considerable attention has been given to the fundamental role of in silico techniques to i) further investigate the spectrum of this secondary metabolite and ii) elucidate the pathways of BEA for its promising therapeutic action.
\end{abstract}

Keywords: Drug repositioning, Molecular modelling, Beauvericin

\section{BEAUVERICIN: A FUNGAL METABOLITE}

Ages ago, before pure chemicals were synthesized, people used natural compounds, for their beneficial effect in traditional medicine and pest management within a harmonized ecosystem. In particular, the secondary metabolites of such natural products are synthesized mainly by bacteria, fungi, and plants [1]. They are molecules of low molecular weight with diverse chemical structures and biological activities. Although they are not required for the development of organisms, they are produced to confer a selective advantage for the organism's survival in nature [2] . Paradoxically, these compounds (or their derivatives) constitute approximately $35 \%$ of the FDA-approved drugs list used by humans during the last two decades to control/cure diseases and/or combat pathogens including bacteria/fungi/viruses [3]. Specifically, recent interest has been noticed in the assessment of fungal metabolites for their wide spam of bioactivities [4]. Beauveria, a cosmopolitan anamorphic genus of soilborne necrotrophic entomopathogenic fungi, is the most studied biological control agent. The global distribution in addition to its broad range of target pests have made Beauveria the most famous entomopathogenic fungi [5]. Its ability to cause diseases in insects was first reported more than 60 years ago. Since that time, Beauveria is the most studied alternative control agent, and currently, its conidia form the basis of several commercial insecticides available in the world trade [6]. The first 
taxonomical characteristic for identifying Beauveria genus was based on the morphology of conidiogenous cells and the conidiogenesis process itself [7]. This traditional approach was however always controversial, but in 2011 the first molecular approach was adopted instead, using a multi-locus phylogeny of Beauveria based on partial sequences of proteincoding genes RPB1, RPB2, TEF, and the nuclear intergenic region, Bloc [8]. Twelve ex-type species of Beauveria genus were described. Among these, B. bassiana was found in different isolates infecting a wide variety of insects and mites including but not limited to Acari, Lepidoptera, Coleoptera, Hymenoptera, Homoptera, Diptera, Hemiptera, Orthoptera $[9,10]$. Despite being considered a generalist fungus with no host preference, $B$. bassiana virulence on its hosts varies considerably among its different strains [11] . Additionally, and in sharp contrast to other entomopathogenic agents, B. bassiana can infect its host by ingestion or by simple contact. The most familiar mode of infection is penetration through the host tegument, however, infections caused by conidial ingestion and penetration through natural orifices were also reported [12]. After cuticle penetration, the host will initiate cellular and/or humoral immunity reactions. The success of fungal infection usually depends on the capability of entomopathogenic fungi to overcome epicuticular fungistatic compounds, cuticle thickness, mineralization, sclerotization, melanization, protease inhibitors, antimicrobial peptides, and cellular/humoral responses [13]. Interestingly, it was also reported that some hypervirulent strains of $B$. bassiana are able to overcome encapsulation and nodule formation by producing secondary metabolites that are able to dampen the host cellular response [14]. BEA is the most famous secondary metabolite produced by many fungi, including but not limited to Beauveria spp. The cyclic hexadepsipeptide molecule belongs to the enniatin antibiotic family [15]. It contains three Dhydroxyisovaleryl and three N-methyl phenylalanyl residues in an alternating sequence (Figure 1). Nevertheless, BEA lacks resemblance with enniatins produced by Fusarium spp. with respect to biological activities. This distinction could be accounted to the nature of the N-methylamino acid [16].

Molecular Formula: $\mathrm{C}_{46} \mathrm{H}_{59} \mathrm{~N}_{3} \mathrm{O}_{9}$ Molecular Weight: $797.99 \mathrm{~g} / \mathrm{mol}$<smiles>CC(C)C(OC(=O)[C@H](Cc1ccccc1)N(C)C(=O)[C@H](Cc1ccccc1)OC(=O)[C@@H](Cc1ccccc1)N(C)C(=O)[C@@H](Cc1ccccc1)N(C)C(=O)[C@H](Cc1ccccc1)C(C)C)C(C)C</smiles>

Figure 1. Chemical structure of beauvericin

\section{Biochemical properties}

Numerous entomopathogenic fungi are known to produce BEA after reaching the hemocoel of their host. It was shown showed that mutant strains of B. bassiana with a knock-out of the bbBeas gene, responsible for the expression of beauvericin, will cause significantly lower virulence against different insect hosts [17]. It was suggested that this metabolite is able to overpower insect cell defenses including, but not limited to, the antimicrobial lipids and phenols, enzyme inhibitors, and proteins [18]. In addition, BEA was found to inhibit the growth of the insect's microbial flora as well as other organisms that may compete for the limited nutrition [19]. Initially, the insecticidal activity of BEA was confirmed against Aedes aegypti, a mosquito that can spread dengue fever, and Calliphora erythrocephala, flies are important in the field of forensic entomology [20]. Since then, 
much more information on the insecticidal activity of BEA has become available. It was demonstrated that BEA can efficiently control the growth of the Colorado potato beetle, Leptinotarsa decemlineata [21]. BEA also significantly reduced the fitness of Schizaphis graminum by increasing the number of abortive embryos produced by treated females [22] . Among 16 fungal metabolites, BEA showed the highest cytotoxicity to lepidopteran (Spodoptera frugiperda) cell line (SF-9) [23]. In addition, studies on the plant bugs Lygus spp. provided insight into a potential role for BEA in controlling them [24]. Concomitantly, the acaricidal activity of BEA has been explored and solid evidence was put forward to support the potential usefulness of BEA as a promising pest management strategy [25]. Under greenhouse conditions, applications of BEA significantly reduced the populations of Tetranychus urticae, and increased strawberry yields while showing no phytotoxicity and ecotoxicological risk. Furthermore, the efficacy of BEA against all developmental stages of the mite Sarcoptes scabiei was recently confirmed paving the way for its usage as a treatment for sarcoptic infections [26].

The above observations are linked directly to the mechanisms by which BEA exerts its effect on the host, which are tightly associated to the molecular characteristics of the molecule. The first hint to these characteristics is the ionophoric nature of BEA which was first demonstrated in a study back in the 1990's where the authors provided evidence that beauvericin is able to transport small ions across cytoplasmic membranes [27]. This observation was further supported by demonstrating that BEA could establish a cation-selective channel after incorporating in the membrane of mammalian cells [28]. The increased intracellular bioavailability of $\mathrm{Ca}^{2+}$ decreases the transmembrane potential of mitochondria, releases cytochrome c, and activates caspase 3, a critical executioner of apoptosis [29]. Consequently, BEA can initiate cell death via calcium-dependent pathways [30]. Furthermore, an increased concentration of BEA could breach the nucleus and alter the DNA [31]. It was proposed that the secondary metabolite can randomly bind to DNA sequences and that the damaged areas could be fragmented by nucleases.

\section{BEA contamination in food commodities}

Associated with its potential use as a beneficial pesticide for humans, BEA was classified as an emerging mycotoxin due to its high occurrence in food commodities including a broad range of food crops such as wheat, rye, oats, barley, and rice [32].Moreover, the mycotoxin is linked to intoxication of eggs [33], dried fruits [34], coffee [35] , medicinal herbs [36], and feed [37]. The amount of BEA present in food has become an important issue for its risk assessment to human and animal health. Strikingly, the presence of BEA was recorded in $90 \%$ of the examined wheat-based products (pasta, cereals, biscuits, and baby formulas) $[38,39]$. The concentration of the mycotoxin within consumed food may range from negligible traces to multiple $\mathrm{mg} / \mathrm{kg}$ [18]. A review on the toxicological relevance of BEA in food considered an acute exposure to the mycotoxin will unlikely cause any concern for human and livestock health despite the potential ionophoric-related effects it has on mammalian cell walls in general [40]. So far and in the absence of adequate toxicological data, risk assessment on the chronic exposure of BEA is still lacking.

\section{BEA IN HUMAN HEALTH AND DISEASES}

In the last few years, BEA has been exploited in the search for new therapeutic options to be used against human pathogens and diseases. Accumulated data from different angles further showed that BEA is being considered a potential candidate for medicinal research due to its broad range of biological properties. The antibacterial activity of BEA has been investigated against gram-negative and positive bacteria of human, animal, and plant interest $[16,17,41]$. The cytotoxic activity against different cancer cell lines was further explored mainly towards the PC-3M (prostate) and MDA-MB-231 (breast) cells [42], and the secondary metabolite was able to significantly reduce CT-26 and KB-3-1-grafted tumors in a murine model [43]. The first antiviral attributes for BEA were examined on 
the human immunodeficiency virus type 1 (HIV-1) where the specificity of the inhibitory effect of the secondary metabolite was mapped to the enzyme (integrase) involved in the integration of the virus into the host genome [44]. Remarkably, this inhibitory concentration was low relative to the cytotoxic concentrations against all tested human cell lines [45]. The inhibitory potential of BEA was comparable to those of baicalein and robinetin which are previously demonstrated as effective antiviral compounds [46]. Furthermore, and along the anti-inflammatory effects of BEA, it was shown to significantly inhibit the nuclear translocation of the NF- $\kappa$ B pathway subunits p65 and p50 within the RAW264.7 cells without inducing cell toxicity [47]. The inhibition of the NF- $\kappa B$ pathway involved in the upregulation of genes encoding cytokines such as interleukin (IL)-1 and tumor necrosis factor (TNF)- $\alpha$, chemokines, and inflammatory mediators such as inducible nitric oxide (NO) synthase (iNOS) (involved in NO production) and cyclooxygenase (involved in prostaglandin E2 secretion) could alleviate the cytokine storm, a dangerous overreaction of the immune system.

A growing body of literature has revealed the synergetic effect of BEA in combination with other compounds. A study conducted by [48] demonstrated the activity of BEA against the yeast Candida albicans. The authors proved that the secondary metabolite could synergize the effect of a low dosage of ketoconazole and could be used in combination to develop new anti-fungal therapeutics. BEA has a significantly higher toxicological effect when combined with deoxynivalenol in mammalian kidney cells [49]. Furthermore, mixed doses of BEA and pesticides (cyflumetofen, bifenazate, or abamectin) significantly suppressed populations of Tetranychus urticae Koch when compared to single-pesticide treatment [50].

\section{In silico modeling and in vitro confirmation for the role of $B E A$ in drug resistance}

In light of accumulated observations in the field and few published reports, BEA could potentiate the activity of drugs. Most importantly, secondary metabolites could restore the sensitivity of certain molecules against multi-drug resistant (MDR) organisms. It was up to the modern in silico techniques to elucidate the pathway of this interesting activity of BEA. Multi-drug resistance is generating considerable interest since it's the major hurdle against treating a wide variety of diseases [51]. The development of resistance is mainly driven by the misuse and the overuse of antimicrobial agents [52]. The emergence and spread of antimicrobial resistance reduces the effectiveness of the available therapeutic arsenal against a wide range of human, animal, and plant pathogens [53]. According to the World Health Organization (2020), the development of resistance is threatening global health and development thus understanding the underlying resistance mechanisms is important to mitigate the growth and dissemination of resistant populations. In addition to chromosomal mutations, development of resistance was mainly attributed to the xenobiotic efflux pumps from different families [54]. The most common group of transmembrane pumps is the ATP-binding cassettes (ABC) transporters that are ubiquitously present in prokaryotes, archaea, and eukaryotes [55]. The inward-facing conformation of $\mathrm{ABC}$ transporters, formed from two bundles of six transmembrane helices, results in a large internal cavity that binds xenobiotics and chemotherapeutic drugs [56]. The switch from the inward- to the outward-facing conformation will transport the substrate to the extracellular environment [55], reducing as a result its bioavailability inside the cell.

To counteract the multi-drug resistant phenotype of Candida albicans, an opportunistic pathogenic yeast, researchers relied on structural base virtual screening to identify modulators of the fungal ABC transporters [57]. The Alignment Mode algorithm of SwissModel was used to build homology models for the ABC transporters Cdr1 and Cdr2, and virtual screening using docking of ZINC15 database was implemented to find structures that could bind to the internal cavity [58] . Interestingly, among 230 million compounds, BEA and its analogues had the best binding affinity to C. albicans' ABC transporters. We have recently corroborated these findings using in silico techniques and demonstrated the 
ability of BEA to neutralize two and four $\mathrm{ABC}$ transporters belonging to $\mathrm{ABCB}$ and $\mathrm{ABCC}$ subfamilies, respectively [50]. The position and conformation of BEA, docked to the binding cavity of the efflux pumps, were compared to those linked to pesticides. Relatively to all controls, BEA recorded the lowest binding affinity [50] (Figure 2). Using computational techniques, it was speculated that BEA was a competitive inhibitor of membranebound proteins and prevents xenobiotic efflux by inhibiting substrate binding, consequently restoring sensitivity. Thus, combinational therapy using BEA and other drugs can be used to overcome multidrug resistance.

A

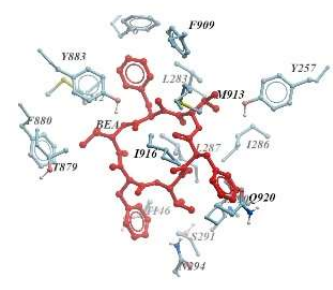

C

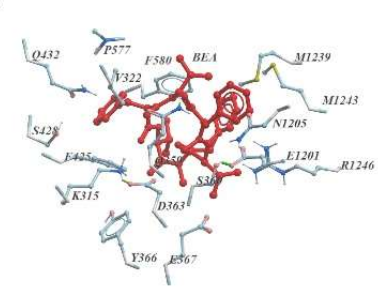

E
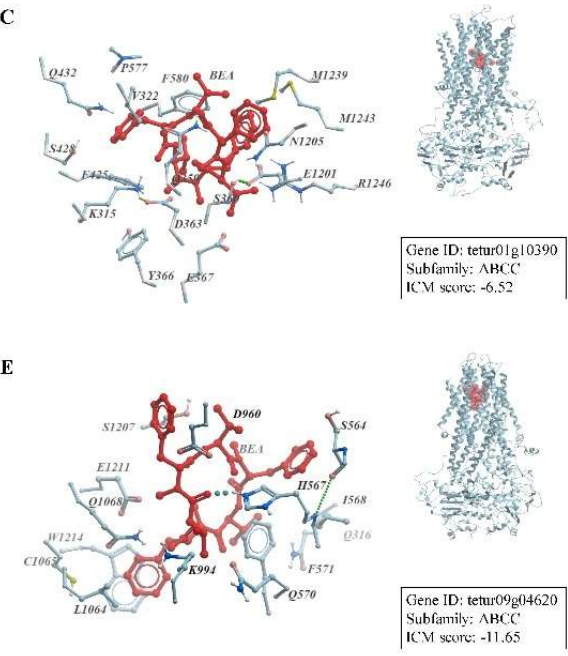

B

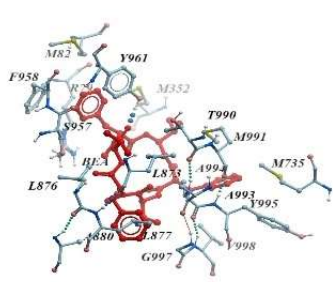

D

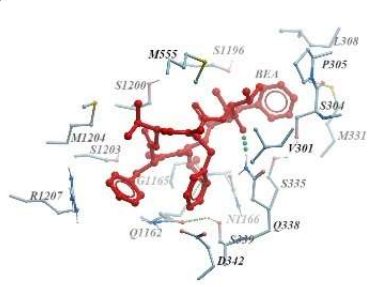

F

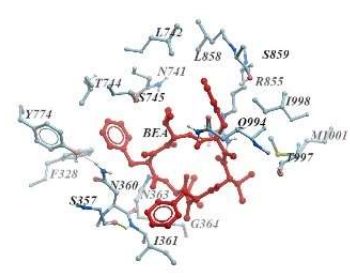

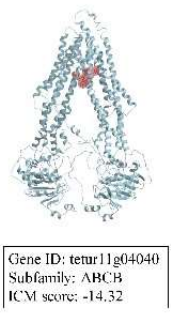

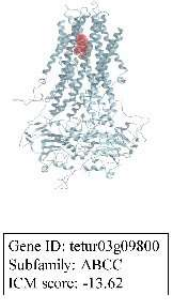

Subfamily: $\mathrm{ABCC}$
IC $\mathrm{BC}$ score: -13.62

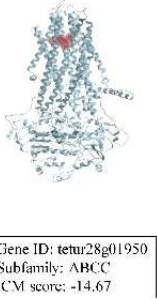

Figure 2. Docking conformations of BEA in ABC transporters (A: tetur11g04030; B: tetur11g04040) and C (C: tetur01g10390; D: tetur03g09800; E: tetur09g04620; F: tetur28g01950).

Beauvericin as a potential anti-viral agent: SARS-CoV-2

In December 2019, a rapidly spreading contagious pneumonia outbreak was first recorded in Wuhan, Hubei province, China. This respiratory disease quickly evolved into a pandemic affecting millions worldwide and referred to as COVID-19 caused by SARSCoV-2 [59]. Multiple studies have confirmed that SARS-CoV-2 is able to attach to host cells via the enzyme angiotensin-converting enzyme 2 (ACE2), which shows enhanced expression in type II alveolar cells of the lungs [60]. In addition, the "cytokine storm" elicited by the virus has channeled the interest of scientists towards reducing the inflammatory response concomitantly with developing antiviral agents for prevention and post-infection therapy. At present, however, the clinical trial registry (ClinicalTrials.gov) encompasses only ongoing clinical trials for existing repurposed FDA-approved drugs. In contrast, researchers in basic laboratory settings are actively testing novel small molecules for their potential therapeutical value. It is therefore imperative in the wake of the challenges caused by the virus to fetch out for such molecules, some of which are actively synthesized in other species or kingdoms. Examples of such potential drugs are secondary metabolites produced by fungi which have been used in agriculture as pesticides and are being 
considered in the medical field as potential therapeutics for different diseases. We thus used the same in silico approach [50] to assess the potential effect of BEA on the different proteins making up the virus (see supplementary material).

\section{Selective and specific docking to viral proteins}

In Numerous drugs as potential inhibitors of the 3CL protease of SARS-CoV-2 were used as positive controls i) chloroquine, an antimalarial drug found to be efficacious in treating COVID-19 infections [61,62] ii) remdesivir effective in preventing replication of SARS-CoV-2 [63] (iii) luteolin, the main flavonoid in honeysuckle [64] that docks perfectly with the main protease of the virus [65] vi) N3 (5-Methylisoxazole-3-carboxylic acid) the inhibitor in complex with the protein crystal structure [66] v) boceprevir a clinically approved anti-hepatitis C drug vi) GC376 the inhibitor of the virus linked to feline infectious peritonitis [67] and the principle components of Kaletra (vii) ritonavir and viii) lopinavir an inhibitor of the SARS-CoV 3CL pro [68]. The most druggable pocket (pocket A: Supplementary Table S1) found in the crystal structure consisted of the inhibitor N3 binding site which demonstrated the high accuracy of the software in predicting "ligandable" orthosteric and allosteric interaction sites. Interestingly, the docking score for BEA against pocket $\mathrm{A}$ of $3 \mathrm{CL}$ protease was the highest $(\mathrm{ICM}$ score $=-7.24 \mathrm{kcal} / \mathrm{mol}$ ) when compared to the positive control ligands (Table 1). Hydrophobic interactions were formed between BEA and H41, M49, F140, L141, H163, M165, L167, P168, D187, R188, Q189, T190, A191. In addition, 1-H bond was established between BEA and E166 (Figure 3). In parallel, BEA recorded the best docking conformation against Pocket B (Supplementary Table S1) relative to the drugs that are in common use. The BEA docked strongly (ICM score: -12.53 $\mathrm{kcal} / \mathrm{mol}$ ) forming $1 \mathrm{H}$-bond with N72, 13 hydrophobic interactions (E14, G15, M17, Q19, A70, G71, V73, P96, K97, N119, G120, S121, and P122), and 5 Van der Waals forces (C16, V18, N28, N95, and Y118) (Figure 3). The ligands used as positive controls presented good docking positions against the main protease of the virus (Pocket A and Pocket B).

In addition to remdesivir and luteolin, ivermectin was docked against the Spike protein of the SARS-CoV-2. Ivermectin showed antiviral activity against SARS-CoV-2-infected Vero-hSLAM cells [69]. Furthermore, data has emerged about the beneficial administration of ivermectin amid COVID-19 infection in hospitalized patients which could account for lower mortality rates [70]. The docking results $(-9.88 \mathrm{kcal} / \mathrm{mol})$ for pocket $\mathrm{A}$, the most "druggable binding site (Supplementary Table S1), revealed that BEA mainly anchors itself to 6VXX through hydrophobic interactions (P225, L226, V227, D228, A520, P521, T523, N544 R557, P579, P561, F562, L582) and one hydrogen bond (K41) (Figure 3). Among all ligands, only luteolin recorded a lower binding affinity when compared to BEA. The BEA docked poorly (Table 1) to the second pocket (Pocket B Supplementary Table S1) identified by the software. We further explored the pocket responsible for the binding of the spike protein to the cell receptor ACE2 [71]. The ICM scores for BEA, remdesivir, luteolin, and ivermectin when docked against the receptor-binding domain were $-1.8,-3.42,-11.42$, and 7.8 , respectively.

MLS001181552, the inhibitor of helicase, as well as astilbin (NPC270578), a plant flavonol, were used as a reference for their demonstrated ability to dock strongly against helicase of SARS-CoV-2 [72]. The SARS-CoV-2 helicase protein recorded a docking score of $-4.82 \mathrm{kcal} / \mathrm{mol}$ with BEA. The anchoring includes i) hydrophobic residues P406, P408, R409, L412, T413, K414, L417, T552, A553, and H554 and ii) a hydrogen bond Y515 to maintain optimal binding (Figure 3). The BEA docked effectively against Pocket A (Supplementary Table S1) however, it docked poorly against the remaining pocket of the SARS-CoV-2 helicase (Table 1). Finally, the binding affinity was the highest amongst all tested ligands (Table 1).

RO-7 and arzanol were used as positive controls because of their abilities to neutralize endoribonucleases [73]. Two different receptor sites were selected for ligand docking (Pocket A and B Supplementary Table S1). The BEA docked poorly against all selected receptors. The best conformation $(-0.07 \mathrm{kcal} / \mathrm{mol}$, Table 1$)$ was recorded against pocket $\mathrm{A}$. 
Based on these findings, BEA will probably not interact with the SARS-CoV-2 endonuclease.

The SARS-CoV-2 RNA-dependent-RNA-polymerase (RdRp) enzyme was also used for docking against BEA, remdesivir, and dATP [72,74]. BEA docked to the active site (Pocket A) of the protein, while showing a relatively poor docking against pocket B (the second most druggable pocket, supplementary table S1). BEA recorded a $-3.05 \mathrm{kcal} / \mathrm{mol}$ score against 7BTF recorded through i) hydrophobic interactions on L172, R249, T252, F321, P322, S255, F396, P461 ii) hydrogen bond on R349 (Figure 3). Despite its relatively low score as compared to Mpro and spike, the binding energy was the highest when compared to the controls.

As for the viral methyltransferase enzyme (PDB:6W4H), the following controls: i) Sadenosyl-L-methionine, the natural ligand of the protein already bound to the $6 \mathrm{~W} 4 \mathrm{H}$ crystal structure ii) sinefungin, a natural nucleoside related to S-adenosyl-L-methionine with demonstrated antiviral activity [75], and iii) 2-(3,4-dihydroxyphenyl)-5,6-dihydroxy-7[(2S,3R,4R,5R,6S)-3,4,5-trihydroxy-6- ethyloxan-2-yl]oxychromen-4-one (NPC226294) [72] were used. The binding receptor site (Pocket A) with the highest "druggability" score (DLID) was the cleft bounding the natural ligand S-adenosyl-L-methionine. BEA docked poorly against this active site of the protein and the binding energy was the highest among all docked ligands. In parallel, BEA docked nicely against pocket B (with the second highest DLID score) (Supplementary Table S1). However, the binding energy was still the lowest when compared to the controls (Table 1). The methyltransferase $(6 \mathrm{~W} 4 \mathrm{H})$ demonstrated a docking score of $-3.94 \mathrm{kcal} / \mathrm{mol}$. BEA bound to the protein mainly across 7 hydrophobic interactions and 2 hydrogen bonds involving G6837, Q4289, P4290, T4292, T4354, T4355, A4357, and N4358 as well as K6836 respectively (Figure 3).

Finally, IcmPocketFinder identified two different druggable pockets in Exoribonuclease (PDB: 5C8T) protein (Supplementary Table S1). 2-(N-Morpholino)-ethanesulfonic acid was earlier identified as an inhibitor of exonucleases [76], and in addition, the natural compound 1-O-(2-Methoxy-4-Acetylphenyl)-6-O-(E-Cinnamoyl)-Beta-D-Glucopyranoside (NPC137813) was recently found as a promising drug to be used against the SARSCoV-2 exoribonuclease [72]. BEA docked poorly to all identified pockets and the recorded binding energy was the highest when compared to both aforementioned controls (Table $1)$.

Awaiting in vitro and pre-clinical experiments, the tests revealed that BEA could bind to different proteins. However, the most striking results that emerged from the data is the low binding energy recorded against the main protease and the spike proteins of the virus. Taken together, BEA may neutralize proteins involved in key developmental stages of SARS-CoV-2. Due to its potential activity on two key proteins, different BEA molecules could have a synergistic effect and thus higher efficacy over existing drugs. Even within one protein, BEA could strongly bind to the orthosteric and allosteric sites. Previous studies showed that BEA has anti-inflammatory activity and it could significantly inhibit the nuclear translocation of the NF- $\kappa$ B pathway subunits p 65 and p50 in RAW264.7 cells without inducing cellular toxicity [47]. The inhibition of the NF- $\kappa$ B pathway was linked to the cytokine storm in severe cases of SARS-CoV-2 [77]. This cellular protection against toxicity when using BEA in vitro was also corroborated in vivo in mice. At this stage of understanding, we believe that BEA may also enhance the therapeutic index of drugs and therefore BEA could be used alone or in combination with other drugs for treating COVID-19 infection. 
a

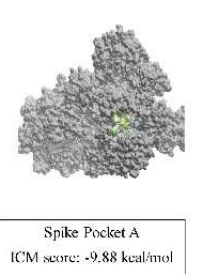

e

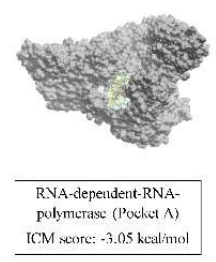

3CL protease Pocket A
ICM score: $-7.24 \mathrm{kcal} / \mathrm{mol}$

c

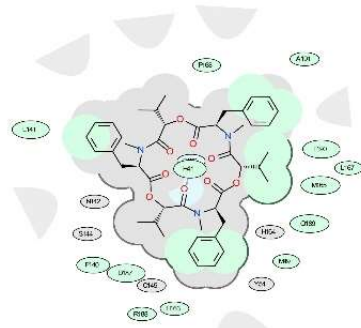

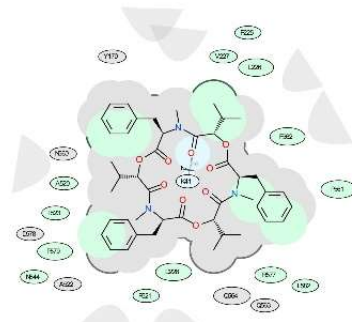

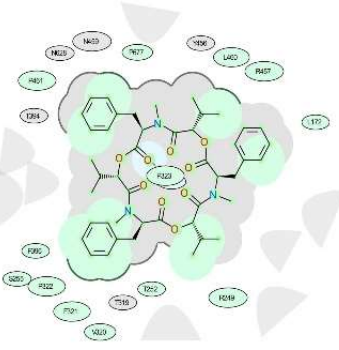

b

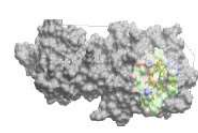

$3 \mathrm{C} \perp$ proleatse Pockel B ICM score: $-12.53 \mathrm{kcal}$ mol

d

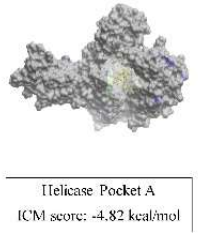

f

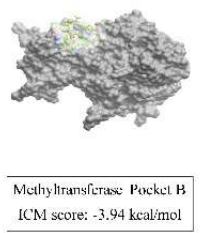

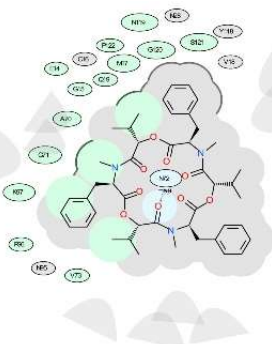

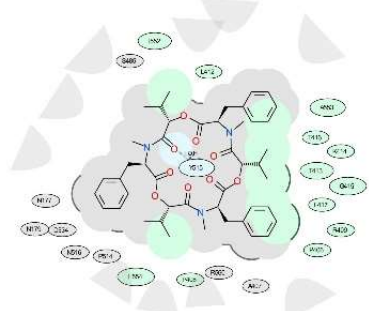

Figure 3. Docking conformations of BEA in SARS-CoV-2. a) 3CL protease (Pocket A) b) 3CL protease (Pocket B) c) Spike Glycoprotein (Pocket A) d) Helicase (Pocket A) e) RNA-dependent-RNA-polymerase (Pocket B) f) Methyltransferase (Pocket B). The ligand 2D representation is showing the contacts between BEA and the different proteins of SARS-CoV-2 (Green shading represents hydrophobic region, blue shading represents hydrogen bond acceptor, white dashed arrows represent hydrogen bonds, grey parabolas represent accessible surface for large areas, grey residues represent "generic" van der Waals contact (non-hydrophobic, non Hbond), size of residue ellipse represents the strength of the contact, 2D distance between residue label and ligand represents proximity). The 3D protein representation is showing the ligand binding pocket on the proteins surface in which these interactions occur (Green: hydrophobic, Red: hydrogen bond acceptor, Blue: hydrogen bond donor).

Table 1. ICM score of beauvericin and different known inhibitors against viral proteins implicated in vital life stages of SARS-CoV-2

\begin{tabular}{|c|c|c|c|}
\hline Protein ID & Pocket ID & Drug & ICM Score $(\mathrm{kcal} / \mathrm{mol})$ \\
\hline \multirow{13}{*}{$\begin{array}{c}\text { 6LU7 } \\
\text { 3CL protease }\end{array}$} & \multirow[t]{9}{*}{ A } & Beauvericin & -7.24 \\
\hline & & Chloroquine & -11.24 \\
\hline & & Remdesivir & -14.37 \\
\hline & & Luteolin & -13.57 \\
\hline & & N3 & -20.46 \\
\hline & & Boceprevir & -10.4 \\
\hline & & GC376 & -20.16 \\
\hline & & Ritonavir & -14.52 \\
\hline & & Lopinavir & -14.35 \\
\hline & \multirow[t]{4}{*}{ B } & Beauvericin & -12.53 \\
\hline & & Chloroquine & -8.04 \\
\hline & & Remdesivir & -7.9 \\
\hline & & Luteolin & -15.15 \\
\hline
\end{tabular}




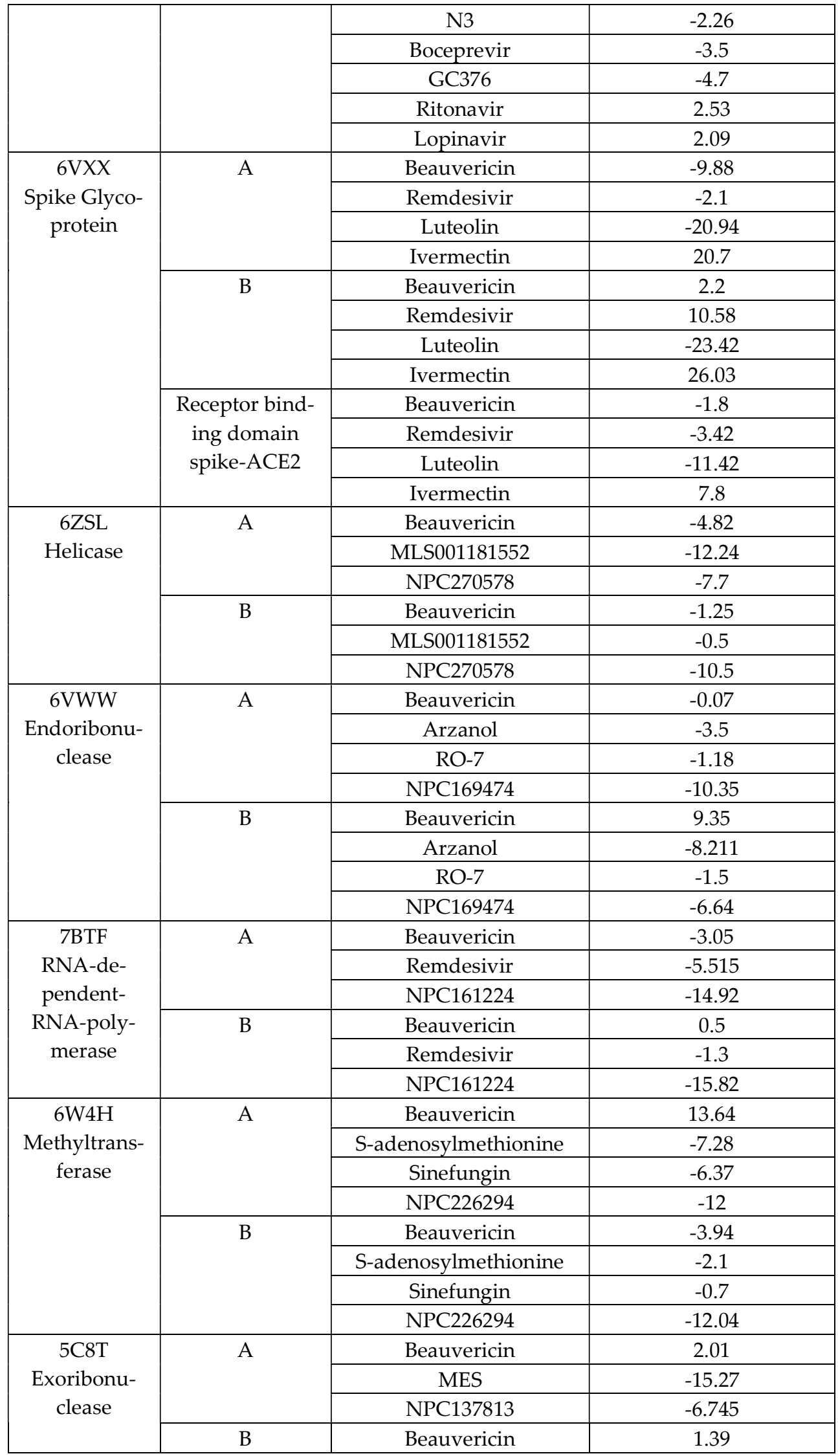




\begin{tabular}{|l|l|c|c|}
\hline \multirow{2}{*}{} & \multirow{2}{*}{} & MES & -14.63 \\
\cline { 3 - 4 } & & NPC137813 & -11.04 \\
\hline
\end{tabular}

\section{Conclusion}

In this review, we described how the advanced in silico strategies guided the drug repositioning of a pesticide to a molecule that inhibits multidrug efflux, providing a powerful strategy to abrogate drug resistance and enhance the efficacy of existing drugs. In addition, we used the most reliable in silico receptor-ligand docking software to determine the likelihood of using a naturally synthesized molecule as an anti-SARS-CoV-2 drug. We thus confirm that modern computational methods have a tremendous impact on the pharmaceutical industry and should be tailored to uncover novel therapeutic options.

Funding: This research received no external funding

Conflicts of Interest: The authors declare no conflict of interest.

\section{References}

1. Barrios-González, J. Secondary metabolites production: physiological advantages in solid-state fermentation. Current developments in biotechnology and bioengineering 2018, 257-283, doi:10.1016/B978-0-444-63990-5.00013-X.

2. Mosunova, O.N.-M., J.C.; Collemare, J. The Biosynthesis of Fungal Secondary Metabolites: From Fundamentals to Biotechnological Applications. Encyclopedia of Mycology 2021, 2, 458-476, doi:10.1016/B978-0-12-809633-8.21072-8.

3. Patridge, E.; Gareiss, P.; Kinch, M.S.; Hoyer, D. An analysis of FDA-approved drugs: natural products and their derivatives. Drug Discov Today 2016, 21, 204-207, doi:10.1016/j.drudis.2015.01.009.

4. Keller, N.P. Fungal secondary metabolism: regulation, function and drug discovery. Nat Rev Microbiol 2019, 17, 167-180, doi:10.1038/s41579-018-0121-1.

5. Rehner, S.A.; Buckley, E. A Beauveria phylogeny inferred from nuclear ITS and EF1-alpha sequences: evidence for cryptic diversification and links to Cordyceps teleomorphs. Mycologia 2005, 97, 84-98, doi:10.3852/mycologia.97.1.84.

6. De Faria, M.R.W.S.P. Mycoinsecticides and Mycoacaricides: A comprehensive list with worldwide coverage and international classification of formulation types. Biological Control 2007, 43, 237-256, doi:10.1016/j.biocontrol.2007.08.001.

7. Sung, J.M.; Lee, J.O.; Humber, R.A.; Sung, G.H.; Shrestha, B. Cordyceps bassiana and Production of Stromata in vitro Showing Beauveria Anamorph in Korea. Mycobiology 2006, 34, 1-6, doi:10.4489/MYCO.2006.34.1.001.

8. Rehner, S.A.; Minnis, A.M.; Sung, G.H.; Luangsa-ard, J.J.; Devotto, L.; Humber, R.A. Phylogeny and systematics of the anamorphic, entomopathogenic genus Beauveria. Mycologia 2011, 103, 1055-1073, doi:10.3852/10-302.

9. Chandler, D.; Davidson, G. Evaluation of entomopathogenic fungus Metarhizium anisopliae against soil-dwelling stages of cabbage maggot (Diptera: Anthomyiidae) in glasshouse and field experiments and effect of fungicides on fungal activity. J Econ Entomol 2005, 98, 1856-1862, doi:10.1093/jee/98.6.1856.

10. Immediato, D.; Camarda, A.; Iatta, R.; Puttilli, M.R.; Ramos, R.A.; Di Paola, G.; Giangaspero, A.; Otranto, D.; Cafarchia, C. Laboratory evaluation of a native strain of Beauveria bassiana for controlling Dermanyssus gallinae (De Geer, 1778) (Acari: Dermanyssidae). Vet Parasitol 2015, 212, 478-482, doi:10.1016/j.vetpar.2015.07.004.

11. Vestergaard, S.C., N.; Keller, S.; Goettel M. Safety of Hyphomycete Fungi as Microbial Control Agents. Environmental Impacts of Microbial Insecticides 2003, 1, 35-62, doi:10.1007/978-94-017-1441-9_3.

12. Aad, G.; Abajyan, T.; Abbott, B.; Abdallah, J.; Abdel Khalek, S.; Abdelalim, A.A.; Abdinov, O.; Aben, R.; Abi, B.; Abolins, M.; et al. Observation of associated near-side and away-side long-range correlations in sqrt $[\mathrm{s}(\mathrm{NN})]=5.02 \mathrm{TeV}$ proton-lead collisions with the ATLAS detector. Phys Rev Lett 2013, 110, 182302, doi:10.1103/PhysRevLett.110.182302.

13. Feng MG, P.T., Khachatourians GG. Production, formulation and application of the entomopathogenic fungus Beauveria bassiana for insect control: current status. Biocontrol Science and technology 1994, 4, 3-34, doi:10.1080/09583159409355309

14. Hung, S.Y.; Boucias, D.G.; Vey, A.J. Effect of Beauveria bassiana and Candida albicans on the cellular defense response of Spodoptera exigua. J Invertebr Pathol 1993, 61, 179-187, doi:10.1006/jipa.1993.1032.

15. Wang, Q.; Xu, L. Beauvericin, a bioactive compound produced by fungi: a short review. Molecules 2012, 17, 2367-2377, doi:10.3390/molecules17032367.

16. Wu, Q.; Patocka, J.; Nepovimova, E.; Kuca, K. A Review on the Synthesis and Bioactivity Aspects of Beauvericin, a Fusarium Mycotoxin. Front Pharmacol 2018, 9, 1338, doi:10.3389/fphar.2018.01338.

17. Xu, Y.; Orozco, R.; Wijeratne, E.M.; Gunatilaka, A.A.; Stock, S.P.; Molnar, I. Biosynthesis of the cyclooligomer depsipeptide beauvericin, a virulence factor of the entomopathogenic fungus Beauveria bassiana. Chem Biol 2008, 15, 898-907, doi:10.1016/j.chembiol.2008.07.011. 
18. Mallebrera, B.; Prosperini, A.; Font, G.; Ruiz, M.J. In vitro mechanisms of Beauvericin toxicity: A review. Food Chem Toxicol 2018, 111, 537-545, doi:10.1016/j.fct.2017.11.019.

19. Gibson, D.M.; Donzelli, B.G.; Krasnoff, S.B.; Keyhani, N.O. Discovering the secondary metabolite potential encoded within entomopathogenic fungi. Nat Prod Rep 2014, 31, 1287-1305, doi:10.1039/c4np00054d.

20. Grove, J.F.P., M. . The insecticidal activity of beauvericin and the enniatin complex. Mycopathologia 1980, 70, 103-105.

21. Gupta, S.M., C.; Hwang, Y.S. Isolation on novel beauvericin analogues from the fungus Beauveria bassiana. Journal of natural products 1996, 58, 733-738.

22. Ganassi, S.; Moretti, A.; Bonvicini Pagliai, A.M.; Logrieco, A.; Agnese Sabatini, M. Effects of beauvericin on Schizaphis graminum (Aphididae). J Invertebr Pathol 2002, 80, 90-96, doi:10.1016/s0022-2011(02)00125-8.

23. Fornelli, F.; Minervini, F.; Logrieco, A. Cytotoxicity of fungal metabolites to lepidopteran (Spodoptera frugiperda) cell line (SF9). J Invertebr Pathol 2004, 85, 74-79, doi:10.1016/j.jip.2004.01.002.

24. Leland, J.E.M., M.R.; Grace,J.A.; Jaronski, S.T.; Ulloa, M.; Park, Y-H.; Plattner, R.D. Strain selection of a fungal entomopathogen, Beauveria bassiana, for control of plant bugs (Lygus spp.) (Heteroptera: Miridae). Biological Control 2005, 35, 104-114, doi:10.1016/j.biocontrol.2005.06.005.

25. Al Khoury, C.G., J.; Nemer, N. Lethal activity of beauvericin, a Beauveria bassiana mycotoxin, against the two-spotted spider mites, Tetranychus urticae Koch. JOURNAL OF APPLIED ENTOMOLOGY 2019, 143, 974-983, doi:10.1111/jen.12684.

26. Al Khoury, C.; Nemer, N.; Nemer, G.; Kurban, M.; Bernigaud, C.; Fischer, K.; Guillot, J. In Vitro Activity of Beauvericin against All Developmental Stages of Sarcoptes scabiei. Antimicrob Agents Chemother 2020, 64, doi:10.1128/AAC.02118-19.

27. Ojcius, D.M.; Zychlinsky, A.; Zheng, L.M.; Young, J.D. Ionophore-induced apoptosis: role of DNA fragmentation and calcium fluxes. Exp Cell Res 1991, 197, 43-49, doi:10.1016/0014-4827(91)90477-c.

28. Kouri, K.; Lemmens, M.; Lemmens-Gruber, R. Beauvericin-induced channels in ventricular myocytes and liposomes. Biochim Biophys Acta 2003, 1609, 203-210, doi:10.1016/s0005-2736(02)00689-2.

29. Jow, G.M.; Chou, C.J.; Chen, B.F.; Tsai, J.H. Beauvericin induces cytotoxic effects in human acute lymphoblastic leukemia cells through cytochrome c release, caspase 3 activation: the causative role of calcium. Cancer Lett 2004, 216, 165-173, doi:10.1016/j.canlet.2004.06.005.

30. Chen, B.F.; Tsai, M.C.; Jow, G.M. Induction of calcium influx from extracellular fluid by beauvericin in human leukemia cells. Biochem Biophys Res Commun 2006, 340, 134-139, doi:10.1016/j.bbrc.2005.11.166.

31. Pocsfalvi, G.; Di Landa, G.; Ferranti, P.; Ritieni, A.; Randazzo, G.; Malorni, A. Observation of non-covalent interactions between beauvericin and oligonucleotides using electrospray ionization mass spectrometry. Rapid Commun Mass Spectrom 1997, 11, 265272, doi:10.1002/(SICI)1097-0231(19970215)11:3<265::AID-RCM848>3.0.CO;2-2.

32. Decleer, M.; Landschoot, S.; De Saeger, S.; Rajkovic, A.; Audenaert, K. Impact of fungicides and weather on cyclodepsipeptideproducing Fusarium spp. and beauvericin and enniatin levels in wheat grains. J Sci Food Agric 2019, 99, 253-262, doi:10.1002/jsfa.9167.

33. Jestoi, M. Emerging fusarium-mycotoxins fusaproliferin, beauvericin, enniatins, and moniliformin: a review. Crit Rev Food Sci Nutr 2008, 48, 21-49, doi:10.1080/10408390601062021.

34. Azaiez, I.G., F.; Sagratini, G.; Mañes, J.; Fernández-Franzón, M. Multi-mycotoxins Analysis in Dried Fruit by LC/MS/MS and a Modified QuEChERS Procedure. Food Analytical Methods 2014, 7, 935-945, doi:10.1007/s12161-013-9785-3.

35. Garcia-Moraleja, A.; Font, G.; Manes, J.; Ferrer, E. Analysis of mycotoxins in coffee and risk assessment in Spanish adolescents and adults. Food Chem Toxicol 2015, 86, 225-233, doi:10.1016/j.fct.2015.10.014.

36. Rychlik, M.; Humpf, H.U.; Marko, D.; Danicke, S.; Mally, A.; Berthiller, F.; Klaffke, H.; Lorenz, N. Proposal of a comprehensive definition of modified and other forms of mycotoxins including "masked" mycotoxins. Mycotoxin Res 2014, 30, 197-205, doi:10.1007/s12550-014-0203-5.

37. Tolosa, J.; Rodriguez-Carrasco, Y.; Ferrer, E.; Manes, J. Identification and Quantification of Enniatins and Beauvericin in Animal Feeds and Their Ingredients by LC-QTRAP/MS/MS. Metabolites 2019, 9, doi:10.3390/metabo9020033.

38. Santini, A.M., G.; Uhlig, S.; Ritieni A. . Fusaproliferin, beauvericin and enniatins: occurrence in food - a review. World Mycotoxin Journal 2012, 5, 71 - 81, doi:10.3920/WMJ2011.1331.

39. Stanciu, O.J., C.; Miere, D.; Loghin, F.; Mañes, J. Occurrence and co-occurrence of Fusarium mycotoxins in wheat grains and wheat flour from Romania. Food Control 2017, 73, 147-155, doi:10.1016j.foodcont.2016.07.042.

40. (CONTAM), E.P.o.C.i.t.F.C. Scientific Opinion on the risks to human and animal health related to the presence of beauvericin and enniatins in food and feed. EFSA JOURNAL 2014, 12, 3802, doi:10.2903/j.efsa.2014.3802.

41. Nilanonta, C.I., M.; Kittakoop, P.; Trakulnaleamsai, S.; Tanticharoen, M.; Thebtaranonth, Y. Precursor-directed biosynthesis of beauvericin analogs by the insect pathogenic fungus Paecilomyces tenuipes Tetrahedron 2002, 58, 3355-3360, doi:10.1016/S00404020(02)00294-6.

42. Zhan, J.; Burns, A.M.; Liu, M.X.; Faeth, S.H.; Gunatilaka, A.A. Search for cell motility and angiogenesis inhibitors with potential anticancer activity: beauvericin and other constituents of two endophytic strains of Fusarium oxysporum. J Nat Prod 2007, 70, 227-232, doi:10.1021/np060394t.

43. Heilos, D.; Rodriguez-Carrasco, Y.; Englinger, B.; Timelthaler, G.; van Schoonhoven, S.; Sulyok, M.; Boecker, S.; Sussmuth, R.D.; Heffeter, P.; Lemmens-Gruber, R.; et al. The Natural Fungal Metabolite Beauvericin Exerts Anticancer Activity In Vivo: A PreClinical Pilot Study. Toxins (Basel) 2017, 9, doi:10.3390/toxins9090258. 
44. Shin, C.G.; An, D.G.; Song, H.H.; Lee, C. Beauvericin and enniatins H, I and MK1688 are new potent inhibitors of human immunodeficiency virus type-1 integrase. J Antibiot (Tokyo) 2009, 62, 687-690, doi:10.1038/ja.2009.102.

45. Olleik, H.; Nicoletti, C.; Lafond, M.; Courvoisier-Dezord, E.; Xue, P.; Hijazi, A.; Baydoun, E.; Perrier, J.; Maresca, M. Comparative Structure-Activity Analysis of the Antimicrobial Activity, Cytotoxicity, and Mechanism of Action of the Fungal Cyclohexadepsipeptides Enniatins and Beauvericin. Toxins (Basel) 2019, 11, doi:10.3390/toxins11090514.

46. Fesen, M.R.; Pommier, Y.; Leteurtre, F.; Hiroguchi, S.; Yung, J.; Kohn, K.W. Inhibition of HIV-1 integrase by flavones, caffeic acid phenethyl ester (CAPE) and related compounds. Biochem Pharmacol 1994, 48, 595-608, doi:10.1016/0006-2952(94)90291-7.

47. Yoo, S.; Kim, M.Y.; Cho, J.Y. Beauvericin, a cyclic peptide, inhibits inflammatory responses in macrophages by inhibiting the NF-kappaB pathway. Korean J Physiol Pharmacol 2017, 21, 449-456, doi:10.4196/kjpp.2017.21.4.449.

48. Tong, Y.; Liu, M.; Zhang, Y.; Liu, X.; Huang, R.; Song, F.; Dai, H.; Ren, B.; Sun, N.; Pei, G.; et al. Beauvericin counteracted multidrug resistant Candida albicans by blocking ABC transporters. Synth Syst Biotechnol 2016, 1, 158-168, doi:10.1016/j.synbio.2016.10.001.

49. Ruiz, M.J.; Franzova, P.; Juan-Garcia, A.; Font, G. Toxicological interactions between the mycotoxins beauvericin, deoxynivalenol and T-2 toxin in CHO-K1 cells in vitro. Toxicon 2011, 58, 315-326, doi:10.1016/j.toxicon.2011.07.015.

50. Al Khoury, C.; Nemer, N.; Nemer, G. Beauvericin potentiates the activity of pesticides by neutralizing the ATP-binding cassette transporters in arthropods. Sci Rep 2021, 11, 10865, doi:10.1038/s41598-021-89622-5.

51. Osagie, E.A.O.S.H.O., E.A. Multiple Drug Resistance- A Fast-Growing Threat. Biomedical Journal of Scientific \& Technical Research 2019, 21, 15715-15726, doi:10.26717/BJSTR.2019.21.003572.

52. Llor, C.; Bjerrum, L. Antimicrobial resistance: risk associated with antibiotic overuse and initiatives to reduce the problem. Ther Adv Drug Saf 2014, 5, 229-241, doi:10.1177/2042098614554919.

53. Li, R.W.S., A.K. Drug Resistance. Brenner's Encyclopedia of Genetics 2013, 418-420, doi:10.1016/B978-0-12-374984-0.00449-6.

54. Chitsaz, M.; Brown, M.H. The role played by drug efflux pumps in bacterial multidrug resistance. Essays Biochem 2017, 61, 127139, doi:10.1042/EBC20160064.

55. Merzendorfer, H. ABC Transporters and Their Role in Protecting Insects from Pesticides and Their Metabolites. Advances in Insect Physiology 2014, 46, 1-72, doi:10.1016/B978-0-12-417010-0.00001-X.

56. Lage, H. ABC-transporters: implications on drug resistance from microorganisms to human cancers. Int J Antimicrob Agents 2003, 22, 188-199, doi:10.1016/s0924-8579(03)00203-6.

57. Choudhury, H.G.; Tong, Z.; Mathavan, I.; Li, Y.; Iwata, S.; Zirah, S.; Rebuffat, S.; van Veen, H.W.; Beis, K. Structure of an antibacterial peptide ATP-binding cassette transporter in a novel outward occluded state. Proc Natl Acad Sci U S A 2014, 111, 91459150, doi:10.1073/pnas.1320506111.

58. Sterling, T.; Irwin, J.J. ZINC 15--Ligand Discovery for Everyone. J Chem Inf Model 2015, 55, 2324-2337, doi:10.1021/acs.jcim.5b00559.

59. Zhu, N.; Zhang, D.; Wang, W.; Li, X.; Yang, B.; Song, J.; Zhao, X.; Huang, B.; Shi, W.; Lu, R.; et al. A Novel Coronavirus from Patients with Pneumonia in China, 2019. N Engl J Med 2020, 382, 727-733, doi:10.1056/NEJMoa2001017.

60. Hoffmann, M.; Kleine-Weber, H.; Schroeder, S.; Kruger, N.; Herrler, T.; Erichsen, S.; Schiergens, T.S.; Herrler, G.; Wu, N.H.; Nitsche, A.; et al. SARS-CoV-2 Cell Entry Depends on ACE2 and TMPRSS2 and Is Blocked by a Clinically Proven Protease Inhibitor. Cell 2020, 181, 271-280 e278, doi:10.1016/j.cell.2020.02.052.

61. Gao, J.; Tian, Z.; Yang, X. Breakthrough: Chloroquine phosphate has shown apparent efficacy in treatment of COVID-19 associated pneumonia in clinical studies. Biosci Trends 2020, 14, 72-73, doi:10.5582/bst.2020.01047.

62. Devaux, C.A.; Rolain, J.M.; Colson, P.; Raoult, D. New insights on the antiviral effects of chloroquine against coronavirus: what to expect for COVID-19? Int J Antimicrob Agents 2020, 55, 105938, doi:10.1016/j.ijantimicag.2020.105938.

63. Mothay, D.; Ramesh, K.V. Binding site analysis of potential protease inhibitors of COVID-19 using AutoDock. Virusdisease 2020, 1-6, doi:10.1007/s13337-020-00585-z.

64. Li, C.; Zang, C.; Nie, Q.; Yang, B.; Zhang, B.; Duan, S. Simultaneous determination of seven flavonoids, two phenolic acids and two cholesterines in Tanreqing injection by UHPLC-MS/MS. J Pharm Biomed Anal 2019, 163, 105-112, doi:10.1016/j.jpba.2018.08.058.

65. Yu, R.; Chen, L.; Lan, R.; Shen, R.; Li, P. Computational screening of antagonists against the SARS-CoV-2 (COVID-19) coronavirus by molecular docking. Int J Antimicrob Agents 2020, 56, 106012, doi:10.1016/j.ijantimicag.2020.106012.

66. Jin, Z.; Du, X.; Xu, Y.; Deng, Y.; Liu, M.; Zhao, Y.; Zhang, B.; Li, X.; Zhang, L.; Peng, C.; et al. Structure of M(pro) from SARSCoV-2 and discovery of its inhibitors. Nature 2020, 582, 289-293, doi:10.1038/s41586-020-2223-y.

67. Fu, L.; Ye, F.; Feng, Y.; Yu, F.; Wang, Q.; Wu, Y.; Zhao, C.; Sun, H.; Huang, B.; Niu, P.; et al. Both Boceprevir and GC376 efficaciously inhibit SARS-CoV-2 by targeting its main protease. Nat Commun 2020, 11, 4417, doi:10.1038/s41467-020-18233-x.

68. Nukoolkarn, V.; Lee, V.S.; Malaisree, M.; Aruksakulwong, O.; Hannongbua, S. Molecular dynamic simulations analysis of ritonavir and lopinavir as SARS-CoV 3CL(pro) inhibitors. J Theor Biol 2008, 254, 861-867, doi:10.1016/j.jtbi.2008.07.030.

69. Caly, L.; Druce, J.D.; Catton, M.G.; Jans, D.A.; Wagstaff, K.M. The FDA-approved drug ivermectin inhibits the replication of SARS-CoV-2 in vitro. Antiviral Res 2020, 178, 104787, doi:10.1016/j.antiviral.2020.104787.

70. Sharun, K.; Dhama, K.; Patel, S.K.; Pathak, M.; Tiwari, R.; Singh, B.R.; Sah, R.; Bonilla-Aldana, D.K.; Rodriguez-Morales, A.J.; Leblebicioglu, H. Ivermectin, a new candidate therapeutic against SARS-CoV-2/COVID-19. Ann Clin Microbiol Antimicrob 2020, 19, 23, doi:10.1186/s12941-020-00368-w. 
71. Lan, J.; Ge, J.; Yu, J.; Shan, S.; Zhou, H.; Fan, S.; Zhang, Q.; Shi, X.; Wang, Q.; Zhang, L.; et al. Structure of the SARS-CoV-2 spike receptor-binding domain bound to the ACE2 receptor. Nature 2020, 581, 215-220, doi:10.1038/s41586-020-2180-5.

72. Naik, B.; Gupta, N.; Ojha, R.; Singh, S.; Prajapati, V.K.; Prusty, D. High throughput virtual screening reveals SARS-CoV-2 multitarget binding natural compounds to lead instant therapy for COVID-19 treatment. Int J Biol Macromol 2020, 160, 1-17, doi:10.1016/j.ijbiomac.2020.05.184.

73. Jones, J.C.; Marathe, B.M.; Lerner, C.; Kreis, L.; Gasser, R.; Pascua, P.N.; Najera, I.; Govorkova, E.A. A Novel Endonuclease Inhibitor Exhibits Broad-Spectrum Anti-Influenza Virus Activity In Vitro. Antimicrob Agents Chemother 2016, 60, 5504-5514, doi:10.1128/AAC.00888-16.

74. Elfiky, A.A. SARS-CoV-2 RNA dependent RNA polymerase (RdRp) targeting: an in silico perspective. J Biomol Struct Dyn 2020, 1-9, doi:10.1080/07391102.2020.1761882.

75. Bouvet, M.; Debarnot, C.; Imbert, I.; Selisko, B.; Snijder, E.J.; Canard, B.; Decroly, E. In vitro reconstitution of SARS-coronavirus mRNA cap methylation. PLoS Pathog 2010, 6, e1000863, doi:10.1371/journal.ppat.1000863.

76. Huang, K.W.; Hsu, K.C.; Chu, L.Y.; Yang, J.M.; Yuan, H.S.; Hsiao, Y.Y. Identification of Inhibitors for the DEDDh Family of Exonucleases and a Unique Inhibition Mechanism by Crystal Structure Analysis of CRN-4 Bound with 2-Morpholin-4ylethanesulfonate (MES). J Med Chem 2016, 59, 8019-8029, doi:10.1021/acs.jmedchem.6b00794.

77. El-Hachem, N.; Eid, E.; Nemer, G.; Dbaibo, G.; Abbas, O.; Rubeiz, N.; Zeineldine, S.; Matar, G.M.; Bikorimana, J.P.; Shammaa, R.; et al. Integrative Transcriptome Analyses Empower the Anti-COVID-19 Drug Arsenal. iScience 2020, 23, 101697, doi:10.1016/j.isci.2020.101697 